\title{
13
}

\section{The Impact of New Capabilities on the Regional Deterrence Architecture in North-East Asia}

Masashi Murano

The development of technology and the new capabilities that make use of it have had a major impact on the security environment and strategy. The 'nuclear revolution' is a major example, which has been supported by the combination of two deterrence concepts: punishment and denial. A strategy that emphasises deterrence by punishment is based on the view that nuclear weapons are 'absolute weapons'-weapons that are too destructive to ever be used for military purposes - and that their role is limited to deterrence by retaliation. ${ }^{1}$ Based on this view, the minimum second-strike (i.e. assured destruction) capability required to destroy critical infrastructure such as an adversary's capital would be enough to deter the adversary, which has the advantage of keeping the cost of deterrent force structure relatively low.

However, there is a fundamental problem with a deterrence strategy focused on retaliation. This is because retaliation occurs only after deterrence has failed - that is, after an adversary has launched a nuclear or non-nuclear attack and changed the status quo-and it does nothing

1 Bernard Brodie, ed., The Absolute Weapon: Atomic Power and World Order (New York: Harcourt, Brace, 1946). 
to prevent the adversary from taking action or limiting damage. From the perspective of escalation control, denial capability at any level of conflict is thus ideal for a deterrent posture. However, developing a denial-based deterrence posture that was fully capable of damage limitation through nuclear and conventional counterforce capabilities and missile defences was impossible during the Cold War.

New technological developments and the current security environment are forcing the United States and its allies to take the development of denial and damage limitation capabilities more seriously than ever before. ${ }^{2}$ Technologically, the advantage of taking an offensive rather than a defensive position is becoming more apparent; however, this also provides a wider range of active denial and damage limitation options than before. This chapter discusses these developments and their implications for alliances in relation to three main areas: deterrence in space, the role of conventional prompt-strike (CPS) capabilities in North-East Asia and the role of low-yield nuclear warheads introduced in the 2018 Nuclear Posture Review (NPR).

\section{Space Systems in Regional Deterrence Architecture}

Since the 1991 Gulf War, ensuring space control has become an essential element of joint operations for US and modern allied forces. Challengers such as China are attempting to take advantage of this vulnerability. In 2007, China destroyed a satellite as part of a test of a kinetic anti-satellite (ASAT) missile modified from the DF-21 medium-range ballistic missile (MRBM), generating a large quantity of uncontrollable debris. As China expands its own use of space, including operation of the Beidou positioning satellite (its version of a global positioning system) and manned space missions, such as its space station, 'hit-to-kill' kinetic counterspace systems such as ASAT missiles and co-orbital killer satellites will present risks to China's own space activities. Therefore, while it continues to develop kinetic ASAT, China is also simultaneously developing a variety of non-kinetic ASAT systems. These include laser

2 The following is an early argument for the importance of damage limitation: Keith B. Payne, Deterrence in the Second Nuclear Age (Lexington: University Press of Kentucky, 1996). 
dazzling against optical sensors of reconnaissance satellites, uplink and downlink jamming and spoofing of satellite signals, as well as cyber attacks against space control systems. ${ }^{3}$

Non-kinetic counterspace capabilities increase the complexity of escalation control. In general, non-kinetic countermeasures are difficult to recognise. This is because it is difficult to determine in real time whether the damage is accidental or intentional, attacks are difficult to attribute and the function of systems may be restored with no lasting damage when the attack stops. Such capabilities can thus easily enable the creation of a grey zone, making the threshold that justifies proportional response ambiguous and deterrence by punishment difficult. Reversible disruptions could also have a lower threshold of use than irreversible damaging attacks. Therefore, to deter such interference, it is necessary not only to strengthen the resiliency of space systems, but also to possess and operate similar counterspace capabilities and conduct dynamic escalation control. In this regard, Japan's decision to acquire countermeasures in the space domain in its 2018 National Defense Program Guidelines is notable and a step in the right direction. ${ }^{4}$

Since ambiguity in Space Situational Awareness (SSA) can lead to delays in decision-making and disruptions to command and control, it is essential to improve SSA, first and foremost through international cooperation, to address these problems. At the same time, it is necessary to strengthen the technological resilience, as well as the political resilience, of space systems to deter interference and disruption to space assets. Technological resilience means dispersing vulnerabilities through enhanced interoperability, miniaturisation and constellation of satellites to maintain mission capabilities and speed up recovery even in the event of an attack. One of these efforts is the Blackjack program led by the US Defense Advanced Projects Agency, which aims to demonstrate how an autonomous small satellite constellation deployed in low Earth orbit can replace the same functions traditionally performed by larger, more expensive satellites deployed in geostationary orbit. ${ }^{5}$ In both areas-SSA and space resilience-allies have the ability to make real contributions through their own efforts.

3 Todd Harrison, Kaitlyn Johnson and Thomas G. Roberts, Space Threat Assessment 2019, CSIS, April 2019, www.csis.org/analysis/space-threat-assessment-2019.

4 Ministry of Defense of Japan, 'National Defense Program Guidelines', 18 December 2018, www.mod.go.jp/en/d_act/d_policy/national.html.

5 Stephen Forbes, 'Blackjack', DARPA, accessed 14September 2021, www.darpa.mil/program/blackjack. 
Conversely, political resilience is an effort to raise the bar for attack by complicating the adversary's strategic calculations. One way to do this is through hosted payloads in which mission equipment is carried as an extra load on board a different country's satellite. For example, the Japanese and US governments exchanged letters under the Mutual Defense Assistance Agreement in December 2020 to carry US SSA sensors on Japan's Quasi-Zenith Satellite System, units 6 and 7, which are scheduled to be operational by the 2023 fiscal year, and to cooperate in improving SSA capabilities. ${ }^{6}$

From the point of view of a potential aggressor, the risk of horizontal escalation arises when attacking a satellite carrying the payload of multiple countries rather than a satellite operated by a single country. Further, if hosted payloads are possible not only between the US and Japan but also between partners that do not necessarily share a common geographic theatre, such as Japan-Australia, Japan-Europe, US-Europe and US-Australia, it could have a cross-regional deterrent effect that is not limited to specific scenarios. For example, if China attempts to disrupt a satellite in the case of a Taiwan contingency, and the target US or Japanese satellite is shared by a European country or Australia, this would complicate China's calculations and may dissuade an attack on the satellite. But similar considerations would also extend to Russian attacks on the same system.

\section{Conventional Prompt-Strike and Deterrence by Denial}

One of the features of today's security environment and military-related technologies is the increase of operational tempo. While the development of command, control, communications, computers, intelligence, surveillance and reconnaissance (C4ISR)—including space systems-and other factors are responsible for this increase in operational tempo, it is also due to the growing impact of long-range, prompt-strike weapons in the region.

6 Ministry of Foreign Affairs of Japan, 'Exchange of Letters on Hosted Payload Cooperation under the Mutual Defense Assistance Agreement between Japan and the United States of America', 15 December 2020, www.mofa.go.jp/mofaj/press/release/press3_000392.html [in Japanese]; 'Japan's Office of National Space Policy Signs Historic MOU with the US Space Force', Space Force Public Affairs, 18 December 2020, www.spaceforce.mil/News/Article/2451728/japans-office-of-nationalspace-policy-signs-historic-mou-with-the-us-space-for/. 
Conventional prompt global strike (CPGS) weapons, as conceived by G. W. Bush's administration in the 2000s, were originally intended to address two related problems: first, to give the US the ability to hit targets across the globe in a short period; second, to provide a strike option that was non-nuclear. This was because, until the introduction of low-yield W76-2 warheads in the 2018 NPR, the only US prompt global strike options were intercontinental ballistic missiles (ICBMs) or submarine-launched ballistic missiles (SLBMs) with high-yield nuclear warheads. However, this challenge has not been fully resolved to date. US CPS programs that are under way have an intermediate range, and do not yet include truly global strike options. ${ }^{7}$ This means that, even if these weapons are deployed in the near future, forward deployment will be essential to their operation. Therefore, the role of allies is critical.

The nature of intermediate-range or theatre-range CPS differs from both CPGS and the low-yield nuclear SLBM. What the low-yield SLBM and previous plans for CPGS have in common is that they are deployed in very small numbers. ${ }^{8}$ Limited numbers of these warheads are intended to help with escalation control, especially against limited nuclear use by Russia, rather than in a counterforce strike disarming campaign against peer competitors such as Russia or China. However, unlike strategic hypersonic weapons, theatre-range hypersonic weapons will be battlefield weapons used for actual warfighting purposes.

These weapons have the potential to significantly change the strategic stability of the region. However, it should not be overlooked that China and North Korea deployed many prompt conventional strike weapons (basically, most ballistic missiles reach hypersonic speeds) before the US. This is important in considering the appropriate combination of strike and defence capabilities that the US and its allies should develop now that the Intermediate-Range Nuclear Forces Treaty is no longer in place.

7 In this program, the US Navy and Army will develop a shared 34.5-inch, two-stage solid rocket motor with a common hypersonic glide body, or C-HGB, but its range is expected to be around 1,400 miles $(2,250 \mathrm{~km})$. The US Air Force's hypersonic glide weapon program, Air-Launched Rapid Response Weapon (ARRW), also has a range of less than 575 miles $(925 \mathrm{~km})$. See Kelley M. Sayler, Hypersonic Weapons: Background and Issues for Congress, Congressional Research Service, 26 April 2021.

8 Department of Defense, Nuclear Posture Review, February 2018, 54, media.defense.gov/2018/ Feb/02/2001872886/-1/-1/1/2018-NUCLEAR-POSTURE-REVIEW-FINAL-REPORT.PDF. 
China has already deployed nearly 1,250 short and medium-range missiles and more than 500 mobile launchers as counter-power projection capabilities, and it would be impossible for the US and its allies to quickly neutralise them if a crisis happened. ${ }^{9}$ On the other hand, China would need more than missile strikes to secure air and maritime superiority over the first island chain in a crisis. Ultimately, that would require continuous deployment of air and naval power. Therefore, to defeat the Chinese theory of victory, forward-deployed CPS weapons should be used to target Chinese air and naval bases and forces, not mobile missile launchers. Ballistic missiles (or hypersonic glide vehicles) are effective for achieving mission-kill against hard targets, even with conventional warheads. For example, if the Chinese Air Force's 3,000-metre class runways are attacked with ballistic missiles at equal intervals, they will be rendered inoperable, weakening China's offensive counter-air capabilities. ${ }^{10}$ If the US and its allies are able to deploy deep-strike capable CPS weapons against mainland China, China would need to invest more in modernising its air defence systems to mitigate the risk. Hence, even short of use, the deployment of these systems would be an effective cost imposition strategy for the US and its allies.

However, the reaction of North Korea to such a deployment will be different from that of China. North Korea is currently estimated to have around 250 mobile missile launchers for short-range ballistic missiles and MRBMs. ${ }^{11}$ Their production rates are uncertain, but it is almost certain that they will be able to produce medium-sized transporter erector launchers domestically, and there is no doubt that their volumes will increase until the 2030s. Also, solid-fuel missiles are comprising an increasing share of the theatre-range missile force, and the time window for identifying, targeting and destroying these systems is becoming very limited. Unlike in the Chinese case, this missile force would be the central target of allied CPS.

9 Department of Defense, Military and Security Developments Involving the People's Republic of China 2020: Annual Report to Congress, September 2020, china.usc.edu/department-defense-militaryand-security-developments-involving-peoples-republic-china-2020.

10 See also the discussion below on post-INF strike options: Masashi Murano, 'The Japan-US Alliance in a Post-INF World: Building an Effective Deterrent in the Western Pacific', nippon.com, 18 December 2019; Masashi Murano, 'The Modality of Japan's Long-Range Strike Options', Texas National Security Review, 1 October 2020.

11 National Air and Space Intelligence Center and Defense Intelligence Ballistic Missile Analysis Committee, Ballistic and Cruise Missile Threat, July 2020, media.defense.gov/2021/ Jan/11/2002563190/-1/-1/1/2020\%20BALLISTIC\%20AND\%20CRUISE\%20MISSILE\%20 THREAT_FINAL_2OCT_REDUCEDFILE.PDF. 
However, it is highly unlikely that North Korea could or would develop and deploy an advanced missile defence system like the one deployed by the US and its allies, or which could be deployed by China, to counter CPS. As the US and its allies are acutely aware, the cost of acquiring and operating mid-course missile defence systems is enormous. Therefore, Pyongyang's strategic portfolio will remain focused on mobile missiles and nuclear weapons.

In short, the deployment ofCPS by the US and its allies will not structurally change North Korea's strategic portfolio. Instead, its increasing number of mobile missiles will make it more costly for the US and its allies to continue investing in missile defence. In other words, the CPS needs to be positioned as a genuine denial and damage limitation option rather than as leverage to change the nature of the confrontation.

Since the 1991 Gulf War, US air-strike campaigns have been conducted primarily with fighter-based strike packages and subsonic cruise missiles, such as Tomahawk. However, it would take around an hour for a subsonic fighter or cruise missile sortie from Japan to reach North Korea. This does not lend itself to strikes that could immediately suppress mobile missiles that are at high launch readiness. Therefore, to suppress North Korea's mobile missiles, the use of ground-launched, medium-range CPS combined with advanced space-based and airborne intelligence, surveillance and reconnaissance assets should be considered. A precision-guided MRBM or hypersonic glide vehicle that could hit North Korea within minutes from Japan might solve the current problems associated with the use of aircraft or subsonic cruise missiles. Nevertheless, given the growing number of North Korea's mobile launchers, such damage limitation strikes might need to be combined with low-yield nuclear options.

\section{Low-Yield Nuclear Weapons in North-East Asia}

The 2018 NPR decided to strengthen the flexibility of US nuclear force structure, especially with the low-yield W76-2 warhead on Trident D5 SLBM. In addition, it foreshadowed the development of a new low-yield and sea-based nuclear cruise missile. The NPR described them 
as serving a deterrent role against Russia's 'escalate to de-escalate' strategy by non-strategic nuclear forces. ${ }^{12}$ Yet these sea-based systems also have a global impact.

Certainly, the visibility of the airborne leg of the nuclear triad (i.e. the strategic bombers) with air-launched cruise missiles is effective as a deterrent signal. In addition, forward-deployable, dual-capable aircraft (DCA) can deliver B61 gravity bombs with low yields, which can provide the essential flexibility for tailored deterrent architecture in North-East Asia. However, as mentioned earlier, given the increase in theatre-range missile threats from North Korea and China, the US and its allies will need to re-evaluate the risks of deployment of these aerial assets to nearby forward bases in Japan, South Korea and even in Guam when military tensions rise. Since US dual-capable stealth assets are hard to detect and intercept in the air, adversaries have an incentive to use their theatre-range strike capabilities against them early in a confrontation. This is because detection and neutralisation have a much higher probability of success while such assets are on the ground.

A sea-based, survivable low-yield nuclear option has different roles and characteristics than air assets. According to the 2018 NPR, these low-yield options are not intended for nuclear warfighting, but to deter adversaries, especially Russian limited nuclear use in a conflict. However, the 2018 NPR emphasises substantial US counterforce capabilities and describes the specific tailored deterrence strategies for each country. As the nuclear forces of China and North Korea are composed mainly of road-mobile systems, it makes sense to include targets such as mobile missiles and their hardened shelters in consideration of the new capabilities, as well as Chinese air and naval forces.

In the current environment, the targets that might need immediate suppression are North Korean nuclear-tipped mobile ballistic missiles. This includes MRBMs such as the Nodong, Scud-ER (able to attack Japan), intermediate-range Hwasong-12 (which puts Guam within range), Hwasong-14/15/16 and North Korea's ICBM. In addition, North Korea has already finished developing several solid-fuel missiles, such as the Pukguksong-2 MRBM. A conflict on the Korean Peninsula would be a very

12 Department of Defense, Nuclear Posture Review. 
challenging situation for Japan, and it is necessary to at least consider all various countermeasures, including a nuclear first-use option to suppress the North Korean mobile missile bases or the missiles themselves.

Even when considering a confrontation with China, low-yield SLBMs have an essential role. According to Indo-Pacific Command (INDOPACOM) estimates, China has already gained a significant temporal advantage over the US in terms of the forces that can be deployed into the Western Pacific in a short time. ${ }^{13}$ Until the US and its allies can deploy sufficient CPS in the region to offset this temporal disadvantage, the prompt-strike capability of low-yield SLBM will also play a supplementary role on the escalation ladder as part of the regional deterrence architecture. Further, despite the growing precision-strike capability of its missile forces, the People's Liberation Army's continued and improved capability to 'hot-swap' conventional and nuclear warheads on the DF-26 suggests they have an 'escalate to de-escalate' strategy with nuclear weapons at the theatre level. Survivable, forward-deployable and prompt low-yield nuclear options are needed to negate China's dangerous confidence in such a strategy.

\section{Three Recommendations for Managing Escalation in North-East Asia}

As discussed in this chapter, new capabilities are essential for appropriate and flexible escalation control in a security environment in which offensive advantage is becoming more prominent. However, effective deterrence using these capabilities also requires close coordination among allies.

First, to build the denial capability needed for deterrence and prevent escalation of the conflict, it is critical that allies work together to accurately gauge the threat, assess their joint capability (with respect to targets, weapon systems, deployment sites, logistical support and so forth), identify any capability gaps that need to be filled and optimise the allocation of roles, missions and capabilities.

13 Mallory Shelbourne, 'US Indo-Pacific Command Wants \$4.68B for New Pacific Deterrence Initiative', USNI News, 2 March 2021, news.usni.org/2021/03/02/u-s-indo-pacific-commandwants-4-68b-for-new-pacific-deterrence-initiative; Chris Dougherty, 'Moving Beyond A2/AD', CNAS, 3 December 2020, www.cnas.org/publications/commentary/moving-beyond-a2-ad. 
Second, allies need to establish a common operational picture and a joint targeting coordination board. To manage the risk of escalation, Japan should be an active and responsible partner in the drafting and implementation of an operational plan detailing when, how and for what targets CPS and counterspace systems would be used. The Japanese Self-Defense Force's active involvement in US operational planning should also reduce the political risks of deploying US CPS systems to Japan.

Third, allies need to improve conventional-nuclear integration in the context of extended deterrence. Unlike during the Cold War, nuclear operations are no longer the responsibility of US regional combatant commands, but of Strategic Command (STRATCOM). Therefore, linking the agenda of the Extended Deterrence Dialogue with the joint operational planning process through the US-Japan Bilateral Planning Committee would seamlessly construct an escalation ladder from the grey zone to the conventional and nuclear domains, leading to more specific nuclear options for the defence of Japan. Based on these plans, it is desirable to conduct regular US-Japan joint exercises that include not only US Forces Korea and INDOPACOM, but also STRATCOM, to test assumptions and improve cooperation. In particular, the risk to forwarddeployed DCA and strategic bombers at a time of crisis, the frequency of deployment of ballistic missile submarines in Guam and the use of lowyield SLBMs against time-sensitive targets, based on the necessity of their use as a prompt means of suppression of hostile missiles, should be a focus of these activities. 
This text is taken from Alliances, Nuclear Weapons and Escalation: Managing Deterrence in the 21st Century, edited by Stephan Frühling and Andrew O'Neil, published 2021 by ANU Press, The Australian National University, Canberra, Australia.

doi.org/10.22459/ANWE.2021.13 